\title{
Laminar Structure of Ptolemaic Graphs and Its Applications
}

\author{
Ryuhei Uehara* $\quad$ Yushi Uno ${ }^{\dagger}$
}

August 29, 2005

\begin{abstract}
Ptolemaic graphs are graphs that satisfy the Ptolemaic inequality for any four vertices. The graph class coincides with the intersection of chordal graphs and distance hereditary graphs. The graph class can also be seen as a natural generalization of block graphs (and hence trees). In this paper, a new characterization of ptolemaic graphs is presented. It is a laminar structure of cliques, and leads us to a canonical tree representation. The tree representation gives a simple intersection model for ptolemaic graphs. The tree representation is constructed in linear time from a perfect elimination ordering obtained by the lexicographic breadth first search. Hence the recognition and the graph isomorphism for ptolemaic graphs can be solved in linear time. Using the tree representation, we also give an $O(n)$ time algorithm for the Hamiltonian cycle problem. The Hamiltonian cycle problem is NP-hard for chordal graphs, and an $O(n+m)$ time algorithm is known for distance hereditary graphs.
\end{abstract}

Keywords: algorithmic graph theory, data structure, Hamiltonian cycle, intersection model, ptolemaic graphs.

\section{Introduction}

Recently, many graph classes have been proposed and studied [3, 13]. Among them, the class of chordal graphs is classic and widely investigated. One of the reasons is that the class has a natural intersection model and hence a concise tree representation; a graph is chordal if and only if it is the intersection graph of subtrees of a tree. The tree representation can be constructed in linear time, and the tree is called a clique tree since each node of the tree corresponds to a maximal clique of the chordal graph (see [23]). Another reason is that the class is characterized by a vertex ordering, which is called a perfect elimination ordering. The ordering can also be computed in linear time, and a typical way to find it is called the lexicographic breadth first search (LBFS) introduced by Rose, Tarjan, and Lueker [22]. The LBFS is also widely investigated as a tool for recognizing several graph classes (see a comprehensive survey by Corneil [8]). Using those characterizations, many efficient algorithms have been established for chordal graphs; to list a few of them, the maximum weighted clique problem, the maximum weighted independent set problem, the minimum coloring problem [12], the minimum maximal independent set problem [11], and so on. There are also parallel algorithms to solve some of these problems efficiently [18].

Distance in graphs is one of the most important topics in algorithmic graph theory. The class of distance hereditary graphs was introduced by Howorka to deal with the distance property called isometric [15]. Some characterizations of distance hereditary graphs are given by Bandelt and Mulder [1], D'Atri and Moscarini [10], and Hammer and Maffray [14]. Especially, Bandelt and Mulder showed that any distance hereditary graph can be obtained from $K_{2}$ by a sequence of extensions called "adding a pendant vertex" and "splitting a vertex." Using the characterizations, many efficient algorithms have been found for distance hereditary graphs $[6,2,5,21,17,7]$. However, the recognition of distance hereditary graphs in linear time is not so simple; Hammer and Maffray's algorithm [14] fails in some cases, and Damiand, Habib, and Paul's algorithm [9] requires to build a cotree in linear time (see [9, Chapter 4] for further

\footnotetext{
*School of Information Science, Japan Advanced Institute of Science and Technology (JAIST), Ishikawa, Japan. uehara@jaist.ac.jp

${ }^{\dagger}$ Department of Mathematics and Information Sciences, Gradate School of Science, Osaka Prefecture University, Osaka, Japan. uno@mi.s.osakafu-u.ac.jp
} 
details), where the cotree can be constructed in linear time by using recent algorithm based on multisweep LBFS approach by Bretscher, Corneil, Habib, and Paul [4].

In this paper, we focus on the class of ptolemaic graphs. Ptolemaic graphs are graphs that satisfy the Ptolemaic inequality $d(x, y) d(z, w) \leq d(x, z) d(y, w)+d(x, w) d(y, z)$ for any four vertices $x, y, z, w$. (The inequality is also known as "Ptolemy" inequality which seems to be more popular. However we use "Ptolemaic," which is stated by Howorka [16].) Howorka showed that the class of ptolemaic graphs coincides with the intersection of the class of chordal graphs and the class of distance hereditary graphs [16]. Hence the results for chordal graphs and distance hereditary graphs can be applied to ptolemaic graphs. On the other hand, the class of ptolemaic graphs is a natural generalization of block graphs, and hence trees (see [26] for the relationships between related graph classes). However, there are relatively few known results specified to ptolemaic graphs. The reason seems that the class of ptolemaic graphs has no useful characterizations from the viewpoint of the algorithmic graph theory.

We propose in this paper a tree representation of ptolemaic graphs which is based on the laminar structure of cliques of a ptolemaic graph. The tree representation also gives a natural intersection model for ptolemaic graphs, which is defined over directed trees. The tree representation can be constructed in linear time for a ptolemaic graph. The construction algorithm can also be modified to a recognition algorithm which runs in linear time. It is worth remarking that the algorithm is quite simple, especially, much simpler than the combination of two recognition algorithms for chordal graphs and distance hereditary graphs. In the construction and the recognition, the ordering of the vertices produced by the LBFS plays an important role. Therefore, our result adds the class of ptolemaic graphs to the list of graph classes that can be recognized efficiently using the LBFS. Moreover, the tree representation is canonical up to isomorphism. Hence, using the tree representation, we can solve the graph isomorphism problem for ptolemaic graphs in linear time. (We note that a clique tree of a chordal graph is not canonical and the graph isomorphism problem for chordal graphs is graph isomorphism complete.)

The tree representation enables us to use the dynamic programming technique for some problems on ptolemaic graphs $G=(V, E)$. It is sure that the Hamiltonian cycle problem is one of most well known NP-hard problem, and it is still NP-hard even for a chordal graph, and that an $O(|V|+|E|)$ time algorithm is known for distance hereditary graphs [17]. Here, we show that the Hamiltonian cycle problem can be solved in $O(|V|)$ time using the technique if a ptolemaic graph is given in the tree representation.

\section{Preliminaries}

The neighborhood of a vertex $v$ in a graph $G=(V, E)$ is the set $N_{G}(v)=\{u \in V \mid\{u, v\} \in E\}$, and the degree of a vertex $v$ is $\left|N_{G}(v)\right|$ and is denoted by $\operatorname{deg}_{G}(v)$. For a subset $U$ of $V$, we denote by $N_{G}(U)$ the set $\{v \in V \mid v \in N(u)$ for some $u \in U\}$. If no confusion can arise we will omit the index $G$. Given a graph $G=(V, E)$ and a subset $U$ of $V$, the induced subgraph by $U$, denoted by $G[U]$, is the graph $\left(U, E^{\prime}\right)$, where $E^{\prime}=\{\{u, v\} \mid u, v \in U$ and $\{u, v\} \in E\}$. Given a graph $G=(V, E)$, its complement is defined by $\bar{E}=\{\{u, v\} \mid\{u, v\} \notin E\}$, and is denoted by $\bar{G}=(V, \bar{E})$. A vertex set $I$ is an independent set if $G[I]$ contains no edges, and then the graph $\bar{G}[I]$ is said to be a clique.

Given a graph $G=(V, E)$, a sequence of the distinct vertices $v_{1}, v_{2}, \ldots, v_{l}$ is a path, denoted by $\left(v_{1}, v_{2}, \ldots, v_{l}\right)$, if $\left\{v_{j}, v_{j+1}\right\} \in E$ for each $1 \leq j<l$. The length of a path is the number of edges on the path. For two vertices $u$ and $v$, the distance of the vertices, denoted by $d(u, v)$, is the minimum length of the paths joining $u$ and $v$. A cycle is a path beginning and ending with the same vertex. A cycle is said to be a Hamiltonian cycle if it visits every vertex in a graph exactly once.

An edge which joins two vertices of a cycle but is not itself an edge of the cycle is a chord of that cycle. A graph is chordal if each cycle of length at least 4 has a chord. Given a graph $G=(V, E)$, a vertex $v \in V$ is simplicial in $G$ if $G[N(v)]$ is a clique in $G$. An ordering $v_{1}, \ldots, v_{n}$ of the vertices of $V$ is a perfect elimination ordering (PEO) of $G$ if the vertex $v_{i}$ is simplicial in $G\left[\left\{v_{i}, v_{i+1}, \ldots, v_{n}\right\}\right]$ for all $i=1, \ldots, n$. Once a vertex ordering is fixed, we denote $N\left(v_{j}\right) \cap\left\{v_{i+1}, \ldots, v_{n}\right\}$ by $N_{>i}\left(v_{j}\right)$. We also use the notions "min" and "max" to denote the first and the last vertices in an ordered set of vertices, respectively. It is known that a graph is chordal if and only if it has a PEO (see [3, Section 1.2] for further details). A typical way of finding a perfect elimination ordering of a chordal graph in linear time is the lexicographic breadth first search (LBFS), which is introduced by Rose, Tarjan, and Lueker [22], and a comprehensive survey is presented by Corneil [8]. 
It is also known that a graph $G=(V, E)$ is chordal if and only if it is the intersection graph of subtrees of a tree $T$ (see [3, Section 1.2] for further details). Let $T_{v}$ denote the subtree of $T$ corresponding to the vertex $v$ in $G$. Then we can assume that each node $c$ in $T$ corresponds to a maximal clique $C$ of $G$ such that $C$ contains $v$ on $G$ if and only if $T_{v}$ contains $c$ on $T$. Such a tree $T$ is called a clique tree of $G$. From a perfect elimination ordering of a chordal graph $G$, we can construct a clique tree of $G$ in linear time [23]. We sometimes unify a node $c$ of a clique tree $T$ with a maximal clique (or a vertex set) $C$ of $G$.

Given a graph $G=(V, E)$ and a subset $U$ of $V$, an induced connected subgraph $G[U]$ is isometric if the distances in $G[U]$ are the same as in $G$. A graph $G$ is distance hereditary if $G$ is connected and every induced path in $G$ is isometric.

A connected graph $G$ is ptolemaic if for any four vertices $u, v, w, x$ of $G, d(u, v) d(w, x) \leq$ $d(u, w) d(v, x)+d(u, x) d(v, w)$. We will use the following characterization of ptolemaic graphs due to Howorka [16]:

Theorem 1 The following conditions are equivalent: (1) $G$ is ptolemaic; (2) $G$ is distance hereditary and chordal; (3) for all distinct nondisjoint maximal cliques $P, Q$ of $G, P \cap Q$ separates $P \backslash Q$ and $Q \backslash P$.

Let $V$ be a set of $n$ vertices. Two sets $X$ and $Y$ are said to be overlapping if $X \cap Y \neq \emptyset, X \backslash Y \neq \emptyset$, and $Y \backslash X \neq \emptyset$. A family $\mathcal{F} \subseteq 2^{V} \backslash\{\{\emptyset\}\}$ is said to be laminar if $\mathcal{F}$ contains no overlapping sets; that is, for any pair of two distinct sets $X$ and $Y$ in $\mathcal{F}$ satisfy either $X \cap Y=\emptyset, X \subset Y$, or $Y \subset X$. Given a laminar family $\mathcal{F}$, we define laminar digraph $\vec{T}(\mathcal{F})=\left(\mathcal{F}, \vec{E}_{\mathcal{F}}\right)$ as follows; $\vec{E}_{\mathcal{F}}$ contains an $\operatorname{arc}(X, Y)$ if and only if $X \subset Y$ and there are no other subset $Z$ such that $X \subset Z \subset Y$, for any sets $X$ and $Y$. We denote the underlying graph of $\vec{T}(\mathcal{F})$ by $T(\mathcal{F})=\left(\mathcal{F}, E_{\mathcal{F}}\right)$. The following two lemmas for the laminar digraph are known (see, e.g., [20, Chapter 2.2]);

Lemma $2 T(\mathcal{F})$ is a forest.

Lemma 3 If a family $\mathcal{F} \subseteq 2^{V}$ is laminar, we have $|\mathcal{F}| \leq 2|V|-1$.

Hence, hereafter, we call $T(\mathcal{F})(\vec{T}(\mathcal{F}))$ a (directed) laminar forest. We regard each maximal (directed) tree in the laminar forest $T(\mathcal{F})(\vec{T}(\mathcal{F}))$ as a (directed) tree rooted at the maximal set, whose outdegree is 0 in $\vec{T}(\mathcal{F})$. We define a label of each node $S_{0}$ in $\vec{T}(\mathcal{F})$, denoted by $\ell\left(S_{0}\right)$, as follows: If $S_{0}$ is a leaf, $\ell\left(S_{0}\right)=S_{0}$. If $S_{0}$ is not a leaf and has children $S_{1}, S_{2}, \ldots, S_{h}, \ell\left(S_{0}\right)=S_{0} \backslash\left(S_{1} \cup S_{2} \cup \cdots \cup S_{h}\right)$. That is, each vertex $v$ in $V$ appears in $\ell(S)$ where $S$ is the minimal set containing $v$. Since $\mathcal{F}$ is laminar, each vertex in $V$ appears exactly once in $\ell(S)$ for some $S \subseteq V$, and its corresponding node is uniquely determined. We note that internal nodes in $\vec{T}(\mathcal{F})$ have a label $\emptyset$ when it is partitioned completely by its subsets in $\mathcal{F}$. (For example, for $V=\{a, b\}$ and $\mathcal{F}=\{X=\{a, b\}, Y=\{a\}, Z=\{b\}\}$, we have $\ell(X)=\{\emptyset\}, \ell(Y)=\{a\}$, and $\ell(Z)=\{b\}$.)

\section{A Tree Representation of Ptolemaic Graphs}

In this section, we show that ptolemaic graphs have a canonical tree representation, and it can be constructed in linear time.

\subsection{A Tree Representation}

For a ptolemaic graph $G=(V, E)$, let $\mathcal{M}(G)$ be the set of all maximal cliques, i.e.,

$$
\mathcal{M}(G):=\{M \mid M \text { is a maximal clique in } G\},
$$

and $\mathcal{C}(G)$ be the set of nonempty vertex sets defined below:

$$
\mathcal{C}(G):=\bigcup_{S \subseteq \mathcal{M}(G)}\left\{C \mid C=\cap_{M \in S} M, C \neq \emptyset\right\} .
$$

Each vertex set $C \in \mathcal{C}(G)$ is a nonempty intersection of some maximal cliques. Hence, $\mathcal{C}(G)$ contains all maximal cliques, and each $C$ in $\mathcal{C}(G)$ induces a clique. We also denote by $\mathcal{L}(G)$ the set $\mathcal{C}(G) \backslash \mathcal{M}(G)$. That is, each vertex set $L \in \mathcal{L}(G)$ is an intersection of two or more maximal cliques, and hence $L$ is a non-maximal clique. The following properties are crucial. 
Theorem 4 Let $G=(V, E)$ be a ptolemaic graph. Let $\mathcal{F}$ be a family of sets in $\mathcal{L}(G)$ such that $\cup_{L \in \mathcal{F}} L \subset$ $M$ for some maximal clique $M \in \mathcal{M}(G)$. Then $\mathcal{F}$ is laminar.

Proof. To derive a contradiction, we assume that $\mathcal{F}$ is not laminar. Then we have two overlapping vertex sets $L_{1}$ and $L_{2}$ which are properly contained in the maximal clique $M$. Let $v, v_{1}, v_{2}$ be vertices in $L_{1} \cap L_{2}$, $L_{1} \backslash L_{2}$, and $L_{2} \backslash L_{1}$, respectively. By definition, there are sets of maximal cliques $M_{1}^{1}, M_{1}^{2}, \ldots, M_{1}^{a}$, $M_{2}^{1}, M_{2}^{2}, \ldots, M_{2}^{b}$ such that $L_{1}=M_{1}^{1} \cap M_{1}^{2} \cap \cdots \cap M_{1}^{a}$ and $L_{2}=M_{2}^{1} \cap M_{2}^{2} \cap \cdots \cap M_{2}^{b}$. Here, if every $M_{1}^{i}$ with $1 \leq i \leq a$ contains $v_{2}$, we have $v_{2} \in L_{1}$. Thus, there is a maximal clique $M_{1}^{i}$ with $v_{2} \notin M_{1}^{i}$. Similarly, there is a maximal clique $M_{2}^{j}$ with $v_{1} \notin M_{2}^{j}$. Let $L$ be $M_{1}^{i} \cap M_{2}^{j}$. Then we have $v_{1}, v_{2} \notin L$ and $v \in L$ (hence $L \neq \emptyset$ ). Therefore $v_{1} \in M_{1}^{i} \backslash L$ and $v_{2} \in M_{2}^{j} \backslash L$. Moreover, since $v_{1}, v_{2}$ are in $M$, $\left\{v_{1}, v_{2}\right\} \in E$. Thus, $L=M_{1}^{i} \cap M_{2}^{j}$ does not separate $M_{1}^{i} \backslash L$ and $M_{2}^{j} \backslash L$, which contradicts Theorem $1(3)$.

Lemma 5 Let $C_{1}, C_{2}$ be any overlapping sets in $\mathcal{C}(G)$ for a ptolemaic graph $G=(V, E)$. Then $C_{1} \cap C_{2}$ separates $C_{1} \backslash C_{2}$ and $C_{2} \backslash C_{1}$.

Proof. Let $C:=C_{1} \cap C_{2}$. By definition of $\mathcal{C}(G), C \in \mathcal{C}(G)$. Let $C_{i}^{\prime}$ be the sets in $\mathcal{C}(G)$ such that $C \subset C_{i}^{\prime} \subset C_{i}$ and there is no other $C^{\prime}$ with $C \subset C^{\prime} \subset C_{i}^{\prime}$ for $i=1,2$. We first observe that $C_{1}^{\prime}$ and $C_{2}^{\prime}$ are overlapping: If $C_{1}^{\prime}=C_{2}^{\prime}$, we have $C_{1}^{\prime}=C_{2}^{\prime} \subseteq C_{1} \cap C_{2}$ which contradicts that $C=C_{1} \cap C_{2}$ and $C \subset C_{1}^{\prime}$. On the other hand, if $C_{1}^{\prime} \subset C_{2}^{\prime}$, we have $C \subset C_{1}^{\prime} \subset C_{2}^{\prime}$ which contradicts the definition of $C_{2}^{\prime}$.

We show that $C$ separates $C_{1}^{\prime}$ and $C_{2}^{\prime}$. Let $M_{i}$ be maximal cliques that contains $C_{i}^{\prime}$ for $i=1,2$ such that $M_{1}$ is overlapping to $C_{2}^{\prime}$ and $M_{2}$ is overlapping to $C_{1}$. Let $C_{c}:=M_{1} \cap M_{2}$. By definition, $C \subseteq C_{c}$. It is sufficient to show that $C=C_{c}$. To derive contradictions, we assume that $v \in C_{c} \backslash C$. We first assume that $v \in C_{1}^{\prime} \backslash C_{2}^{\prime}$. In the case, since $M_{2}$ and $C_{1}^{\prime}$ are overlapping, $\mathcal{C}$ contains a set $C^{\prime \prime}$ with $\left(C_{1}^{\prime} \cap C_{2}^{\prime}\right) \subset\left(\left(C_{1}^{\prime} \cap C_{2}^{\prime}\right) \cup\{v\}\right) \subseteq C^{\prime \prime} \subset C_{1}^{\prime}$, which is a contradiction. Thus, we have $v \notin C_{1}^{\prime}$ and $v \notin C_{2}^{\prime}$.

By definition of $C_{1}^{\prime}$, there are maximal cliques $M_{1}^{1}, M_{1}^{2}, \ldots, M_{1}^{k}$ such that $C_{1}^{\prime}=\cap_{i=1}^{k} M_{1}^{i}$. Since $v \notin C_{1}^{\prime}$, there is at least one maximal clique $M_{1}^{i}$ with $v \notin M_{1}^{i}$. Similarly, there is at least one maximal clique $M_{2}^{j}$ with $C_{2}^{\prime} \subseteq M_{2}^{j}$ and $v \notin M_{2}^{j}$. However, $C_{1}^{\prime} \subseteq M_{1}^{i}, C_{2}^{\prime} \subseteq M_{2}^{j}$, and $v \notin M_{1}^{i} \cup M_{2}^{j}$ imply that $M_{1}^{i} \backslash M_{2}^{j}$ and $M_{2}^{j} \backslash M_{1}^{i}$ are connected by $v$. This is a contradiction to Theorem 1(3). Hence $M_{1}^{i} \cap M_{2}^{j}=M_{1} \cap M_{2}=C_{1}^{\prime} \cap C_{2}^{\prime}=C_{1} \cap C_{2}$, and it is a separator.

Now we define a directed graph $\vec{T}(\mathcal{C}(G))=(\mathcal{C}(G), A(G))$ for a given ptolemaic graph $G=(V, E)$ as follows: two nodes $C_{1}, C_{2} \in \mathcal{C}(G)$ are joined by an arc $\left(C_{1}, C_{2}\right)$ if and only if $C_{1} \subset C_{2}$ and there is no other $C$ in $\mathcal{C}(G)$ such that $C_{1} \subset C \subset C_{2}$. We denote by $T(\mathcal{C}(G))$ the underlying graph of $\vec{T}(\mathcal{C}(G)$ ).

Theorem 6 A graph $G=(V, E)$ is ptolemaic if and only if the graph $T(\mathcal{C}(G))$ is a tree.

Proof. We first assume that $G$ is ptolemaic and show that $T(\mathcal{C}(G))$ is a tree. It is not difficult to see that $T(\mathcal{C}(G))$ is connected. Thus, to derive contradictions, we assume that $T(\mathcal{C}(G))$ contains a cycle $\left(C_{1}, C_{2}, \ldots, C_{k}, C_{1}\right)$, which is a minimal cycle without chords on $T(\mathcal{C}(G))$. Since $C_{1} \subset C_{2} \subset \cdots \subset C_{k} \subset$ $C_{1}$ (or vice versa) is impossible, there is a node $C_{a}$ with $C_{a-1} \supset C_{a} \subset C_{a+1}$ for some $a$. Without loss of generality, we assume that $\left|C_{a}\right|$ is the smallest among such vertex sets on the cycle. Let $C_{x}$ and $C_{y}$ be the nodes on the cycle such that $C_{x-1} \subset C_{x} \supset C_{x+1} \supset \cdots \supset C_{a-1} \supset C_{a} \subset C_{a+1} \subset \cdots \subset$ $C_{y-1} \subset C_{y} \supset C_{y+1}$. It is not difficult to see that $C_{a-1}$ and $C_{a+1}$, and hence $C_{x}$ and $C_{y}$ are overlapping. Thus, by Lemma 5, $C_{a}$ separates $C_{x} \backslash C_{y}$ and $C_{y} \backslash C_{x}$. Since $C_{a}$ is a separator, we let $G_{x}$ and $G_{y}$ be the connected components that contain $C_{x} \backslash C_{y}$ and $C_{y} \backslash C_{x}$ on $G\left[V \backslash C_{a}\right]$, respectively.

Now we consider the path $\mathrm{P}=\left(C_{x}, C_{x-1}, C_{x-2}, \ldots, C_{y+2}, C_{y+1}, C_{y}\right)$ which does not contain $C_{a}$. However, since $C_{a}$ is a separator, $\mathrm{P}$ contains at least one vertex set $C_{b}$ in $\mathcal{C}$ with $C_{a} \cap C_{b} \neq \emptyset$. If $\left(C_{x} \cap C_{b}\right) \backslash C_{a} \neq \emptyset$ and $\left(C_{y} \cap C_{b}\right) \backslash C_{a} \neq \emptyset, C_{x} \backslash C_{y}$ and $C_{y} \backslash C_{x}$ are connected on $G\left[V \backslash C_{a}\right]$ since $C_{b}$ is a clique. Hence each $C_{b}$ with $C_{a} \cap C_{b} \neq \emptyset$ satisfies $\left(C_{x} \cap C_{b}\right) \backslash C_{a}=\emptyset$ or $\left(C_{y} \cap C_{b}\right) \backslash C_{a}=\emptyset$. Since $\mathrm{P}$ connects $G_{x}$ and $G_{y}$ through the separator $C_{a}$, we have at least two vertex sets $C_{b}$ and $C_{b}^{\prime}$ such that $\left(C_{y} \cap C_{b}\right) \backslash C_{a}=\emptyset$ and $\left(C_{x} \cap C_{b}^{\prime}\right) \backslash C_{a}=\emptyset$. Moreover, since $C_{a}$ separates $G_{x}$ and $G_{y}$, we have $C_{b} \cap C_{b}^{\prime} \subseteq C_{a}$. If $C_{b} \cap C_{b}^{\prime} \subset C_{a}, \mathrm{P}$ contains smaller separator than $C_{a}$. Thus $C_{b} \cap C_{b}^{\prime}=C_{a}$. Then $\mathrm{P}$ has to contain $C_{a}$ between $C_{b}$ and $C_{b}^{\prime}$, which contradicts the minimality of the cycle.

Therefore, $T(\mathcal{C}(G))$ is a tree.

It is easy to see that $G$ is ptolemaic if $T(\mathcal{C}(G))$ is a tree; for each pair of distinct nondisjoint maximal cliques $M_{1}$ and $M_{2},\left(M_{1} \cap M_{2}\right)$ separates $T(\mathcal{C}(G))$, and hence $G$. 
Hereafter, given a ptolemaic graph $G=(V, E)$, we call $T(\mathcal{C}(G))(\vec{T}(\mathcal{C}(G)))$ a (directed) clique laminar tree of $G$. We extend the label of a laminar forest to the directed clique laminar tree naturally: Each node $C_{0}$ in $\mathcal{C}(G)$ has a label $\ell\left(C_{0}\right):=C_{0} \backslash\left(C_{1} \cup C_{2} \cup \cdots \cup C_{h}\right)$, where $\left(C_{i}, C_{0}\right)$ is an arc on $\vec{T}(\mathcal{C}(G))$ for $1 \leq i \leq h$. Intuitively, we additionally define the label of a maximal clique as follows; the label of a maximal clique is the set of vertices which are not contained in any other maximal cliques. We note that for each vertex in $G$ its corresponding node in $T(\mathcal{C}(G))$ is uniquely determined by maximal cliques. Therefore, we can define the mapping from each vertex to the vertex set in $\mathcal{C}$ in $T(\mathcal{C}(G))$ : We denote by $C(v)$ the clique $C$ with $v \in \ell(C)$. When we know whether $C(v)$ is in $\mathcal{M}$ or $\mathcal{L}$, we specify it by writing $C_{M}(v)$ or $C_{L}(v)$. An example is given in Figure 2. In Figure 2, each single rectangle represents a non-maximal clique, each double rectangle represents a maximal clique, and each rectangle contains its label. We also note that from $\vec{T}(\mathcal{C}(G))$ with labels, we can reconstruct the original ptolemaic graph uniquely up to isomorphism. That is, two ptolemaic graphs $G_{1}$ and $G_{2}$ are isomorphic if and only if labeled $\vec{T}\left(\mathcal{C}\left(G_{1}\right)\right)$ is isomorphic to labeled $\vec{T}\left(\mathcal{C}\left(G_{2}\right)\right)$.

Intuitively, a clique laminar tree subdivides a clique tree of a chordal graph. For a chordal graph, maximal cliques are joined in a looser way in the sense that a clique tree for a chordal graph is not always uniquely determined up to isomorphism. The clique laminar tree subdivides the relationships between maximal cliques by using their laminar structure.

The following properties of $\vec{T}(\mathcal{C}(G))$ is easy to see, and useful from the algorithmic point of view:

Corollary 7 If $G$ is a ptolemaic graph, we have the following: (1) For each maximal clique $M$ in $\mathcal{M}(G)$, $\ell(M)$ consists of simplicial vertices in $M$. (2) The vertices in a maximal clique $M$ in $\mathcal{M}(G)$ induce a maximal directed subtree of $\vec{T}(\mathcal{C}(G))$ rooted at the node $M$. (3) Each leaf in $T(\mathcal{C}(G))$ corresponds to a maximal clique in $\mathcal{M}(G)$.

It is well known that a graph is chordal if and only if it is the intersection graph of subtrees of a tree. By Theorem 6, we obtain an intersection model for ptolemaic graphs as follows:

Corollary 8 Let $\vec{T}$ be any directed graph such that its underlying graph $T$ is a tree. Let $\mathcal{T}$ be any set of subtrees $\overrightarrow{T_{v}}$ such that $\overrightarrow{T_{v}}$ consists of a root $C$ and all vertices reachable from $C$ in $\vec{T}$. Then the intersection graph over $\mathcal{T}$ is ptolemaic. On the other hand, for any ptolemaic graph, there exists such an intersection model.

Proof. The directed clique laminar tree $\vec{T}(\mathcal{C}(G))$ is the base directed graph of the intersection model. For each $v \in V$, we define the root $C$ such that $v \in \ell(C)$.

\subsection{A Linear Time Construction of Clique Laminar Trees}

The main theorem in this section is the following:

Theorem 9 Given a ptolemaic graph $G=(V, E)$, the directed clique laminar tree $\vec{T}(\mathcal{C}(G))$ can be constructed in $O(|V|+|E|)$ time.

We will make the directed clique laminar tree $\vec{T}(\mathcal{C}(G))$ by separating the vertices in $G$ into the vertex sets in $\mathcal{C}(G)=\mathcal{M}(G) \cup \mathcal{L}(G)$.

We first compute (and fix) a perfect elimination ordering $v_{1}, v_{2}, \ldots, v_{n}$ by the LBFS. The outline of our algorithm is similar to the algorithm for constructing a clique tree for a given chordal graph due to Spinrad in [23]. For each vertex $v_{n}, v_{n-1}, \ldots, v_{2}, v_{1}$, we add it into the tree and update the tree. For the current vertex $v_{i}$, let $v_{j}:=\min \left\{N_{>i}\left(v_{i}\right)\right\}$. Then, in Spinrad's algorithm [23], there are two cases to consider: $N_{>i}\left(v_{i}\right)=C\left(v_{j}\right)$ or $N_{>i}\left(v_{i}\right) \subset C\left(v_{j}\right)$. The first case is easy; just add $v_{i}$ into $C\left(v_{j}\right)$. In the second case, Spinrad's algorithm adds a new maximal clique $C\left(v_{i}\right)$ that consists of $N_{>i}\left(v_{i}\right) \cup\left\{v_{i}\right\}$. However, in our algorithm, involved case analysis is required. For example, in the latter case, the algorithm have to handle three vertex sets; two maximal cliques $\left\{v_{i}\right\} \cup N_{>i}\left(v_{i}\right)$ and $C\left(v_{j}\right)$ together with one vertex set $N_{>i}\left(v_{i}\right)$ shared by them. In this case, intuitively, our algorithm makes three distinct sets $C_{M}$ with $\ell\left(C_{M}\right)=\left\{v_{i}\right\}, C_{L}$ with $\ell\left(C_{L}\right)=N_{>i}\left(v_{i}\right)$, and $C$ with $\ell(C)=C\left(v_{j}\right) \backslash N_{>i}\left(v_{i}\right)$, and adds two $\operatorname{arcs}\left(C_{L}, C_{M}\right)$ and $\left(C_{L}, C\right)$; this means that $v_{i}$ is in $C_{M}=N_{>i}\left(v_{i}\right) \cup\left\{v_{i}\right\}, C$ is a clique $C\left(v_{j}\right)$, and $C_{L}$ is the vertex set shared by $C_{M}$ and $C$. However, our algorithm has to handle more complicated cases since the set $C\left(v_{j}\right)$ (and hence $N_{>i}\left(v_{i}\right)$ ) can already be partitioned into some vertex sets. 


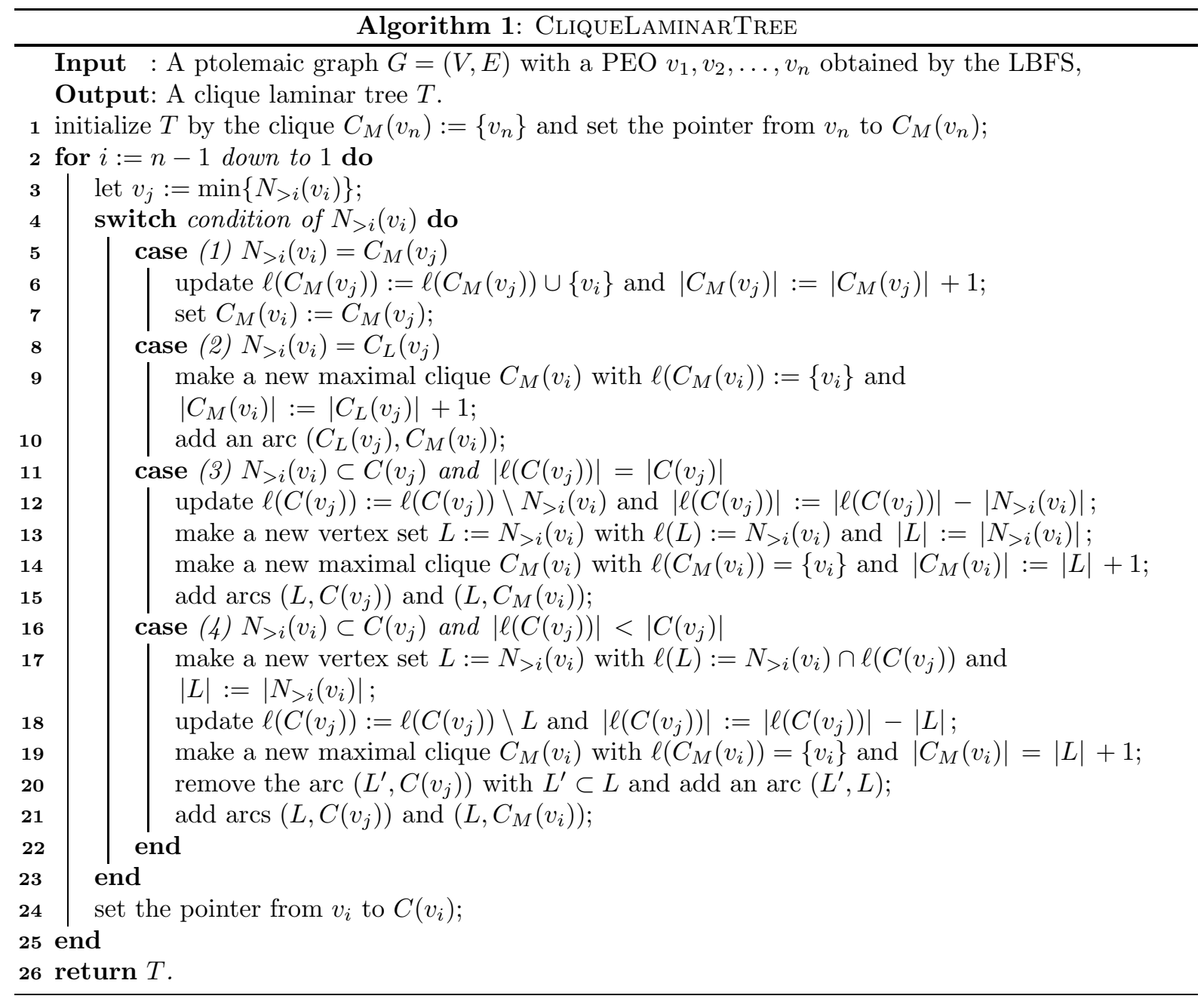

Figure 1: A linear time algorithm for the clique laminar tree $T$ of a ptolemaic graph $G=(V, E)$.

In $\vec{T}(\mathcal{C}(G)$ ), each node $C$ stores $\ell(C)$. Hence each vertex in $G$ appears exactly once in the tree. To represent it, each vertex $v$ has a pointer to the node $C(v)$ in $\mathcal{C}(G)=\mathcal{M}(G) \cup \mathcal{L}(G)$. The detail of the algorithm is described as CliqueLAminarTREe shown in Figure 1, and an example of the construction is depicted in Figure 2. In Figure 2, the left-hand graph gives a ptolemaic graph, and the right-hand trees are clique laminar trees constructed (a) after adding the vertices 16,15,14,13,12,11, (b) after adding the vertices $16,15,14,13,12,11,10$, (c) after adding the vertices $16,15,14,13,12,11,10,9,8$, and (d) after adding all the vertices. We show the correctness and a complexity analysis of the algorithm.

We will use the following property of a PEO found by the LBFS of a chordal graph:

Lemma 10 Let $v_{1}, v_{2}, \ldots, v_{n}$ be a PEO found by the LBFS. Then $i<j$ implies $\max \left\{N\left(v_{i}\right)\right\} \leq$ $\max \left\{N\left(v_{j}\right)\right\}$.

Proof. Let $v_{k}$ be $\max \left\{N\left(v_{i}\right)\right\}$. If $v_{k}$ is a neighbor of $v_{j}$, we have done. Hence we assume that $v_{k} \notin N\left(v_{j}\right)$. Then Theorem 1 in [8] implies that $v_{j}$ should have a neighbor $v_{k^{\prime}}$ with $k^{\prime}>k$.

We assume that Algorithm CliqueLaminarTree is going to add $v_{i}$, and let $v_{j}:=\min \left\{N_{>i}\left(v_{i}\right)\right\}$. We will show that all possible cases are listed, and in each case, CLIQueLAminarTreE correctly manages the nodes in $\mathcal{C}(G)$ and their labels in $O\left(\operatorname{deg}\left(v_{i}\right)\right)$ time. The following lemma drastically decreases the number of possible cases, and simplifies the algorithm.

Lemma 11 Let $v_{k}$ be $\max \left\{N_{>i}\left(v_{i}\right)\right\}$. We moreover assume that the set $N_{>i}\left(v_{i}\right)$ has already been divided into some distinct vertex sets $L_{1}, L_{2}, \ldots, L_{h}$. Then, there is an ordering of the sets such that $v_{k} \in L_{1} \subset$ $L_{2} \subset \cdots \subset L_{h}$. 

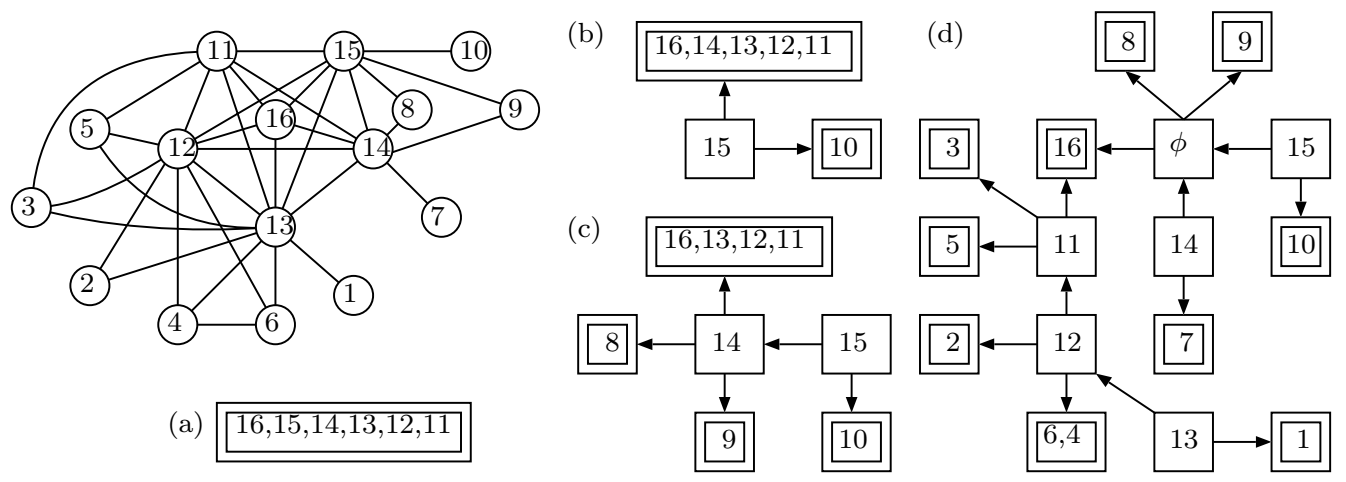

Figure 2: A ptolemaic graph and its clique laminar tree.

Proof. We first observe that $G\left[\left\{v_{i}, v_{i+1}, \ldots, v_{n}\right\}\right]$ is ptolemaic if $G$ is ptolemaic since any vertex induced subgraph of a chordal graph is chordal, and any vertex induced subgraph of a distance hereditary graph is distance hereditary.

We assume that there is a vertex set $L \subset N_{>i}\left(v_{i}\right)$ such that $L$ does not contain $v_{k}$. Then, there is a vertex $v_{i^{\prime}}$ with $i^{\prime}>i$ that makes the vertex set $L$ before $v_{i}$. Since $\left\{v_{i^{\prime}}, v_{k}\right\} \notin E$, by Lemma $10, v_{i^{\prime}}$ has another neighbor $v_{k^{\prime}}$ with $k^{\prime}>k$. By the property of the LBFS, it is easy to see that $G\left[\left\{v_{k}, \ldots, v_{n}\right\}\right]$ is connected. Let $M_{i}$ be a maximal clique $\left\{v_{i}\right\} \cup N_{>i}\left(v_{i}\right)$, and $M_{i^{\prime}}$ be a maximal clique that contains $\left\{v_{i^{\prime}}\right\} \cup L$. Then, $M_{i} \cap M_{i^{\prime}}=L$ which contains no vertex in $G\left[\left\{v_{k}, \ldots, v_{n}\right\}\right]$. On the other hand, we have $\left\{v_{i}, v_{k}\right\},\left\{v_{i^{\prime}}, v_{k^{\prime}}\right\} \in E$. Hence, $M_{i} \cap M_{i^{\prime}}$ does not separate $M_{i} \backslash M_{i^{\prime}}$ and $M_{i^{\prime}} \backslash M_{i}$. Therefore $G\left[\left\{v_{i}, v_{i+1}, \ldots, v_{n}\right\}\right]$ is not ptolemaic by Theorem 1(3), which is a contradiction. Thus we have $v_{k} \in L$, and hence, all the vertex sets $L_{1}, L_{2}, \ldots, L_{h}$ contain $v_{k}$. The vertex set $N_{>i}\left(v_{i}\right)$ is contained in a maximal clique in the ptolemaic graph $G\left[\left\{v_{i}, v_{i+1}, \ldots, v_{n}\right\}\right]$. Hence by Theorem $4, L_{1}, L_{2}, \ldots, L_{h}$ are laminar. Therefore, we have $v_{k} \in L_{1} \subset L_{2} \subset \cdots \subset L_{h}$ for some suitable ordering.

Proof of Theorem 9. (Sketch.) Since the graph $G$ is chordal and the vertices are ordered in a perfect elimination ordering, $N_{>i}\left(v_{i}\right)$ induces a clique. By Lemma 11, we have three possible cases; (a) $N_{>i}\left(v_{i}\right)=$ $C\left(v_{j}\right)$, (b) $N_{>i}\left(v_{i}\right) \subset C\left(v_{j}\right)$ and there are no vertex sets in $N_{>i}\left(v_{i}\right)$, and (c) $N_{>i}\left(v_{i}\right) \subset C\left(v_{j}\right)$ and there are vertex sets $L_{1} \subset L_{2} \subset \cdots \subset L_{h} \subset N_{>i}\left(v_{i}\right)$. In the last case, we note that $L_{h} \neq N_{>i}\left(v_{i}\right)$; otherwise, we have $v_{j} \in L_{h}$, or consequently, $L_{h}=C\left(v_{j}\right)=N_{>i}\left(v_{i}\right)$, which is case (a).

(a) $N_{>i}\left(v_{i}\right)=C\left(v_{j}\right)$ : We have two subcases; $C\left(v_{j}\right)$ is a maximal clique (i.e. $\left.N_{>i}\left(v_{i}\right)=C_{M}\left(v_{j}\right)\right)$ or $C\left(v_{j}\right)$ is a non-maximal clique (i.e. $\left.N_{>i}\left(v_{i}\right)=C_{L}\left(v_{j}\right)\right)$. In the former case, we just update $C_{M}\left(v_{j}\right)$ by $C_{M}\left(v_{j}\right) \cup\left\{v_{i}\right\}$. This is case (1) in CliqueLaminarTree. In the latter case, there are other vertex set that contains $C_{L}\left(v_{j}\right)$ as a subset. Thus we add a new maximal clique $C_{L}\left(v_{j}\right) \cup\left\{v_{i}\right\}$. More precisely, we add a new node $C_{M}\left(v_{i}\right)$ with $\ell\left(C_{M}\left(v_{i}\right)\right)=\left\{v_{i}\right\}$ and $\left|C_{M}\left(v_{i}\right)\right|=\left|C_{L}\left(v_{j}\right)\right|+1$, and a new arc $\left(C_{L}\left(v_{j}\right), C_{M}\left(v_{i}\right)\right)$. This is done in case (2) of CliqueLaminarTree. We can check if $N_{>i}\left(v_{i}\right)=C\left(v_{j}\right)$ by checking if $\left|N_{>i}\left(v_{i}\right)\right|=\left|C\left(v_{j}\right)\right|$ in $O(1)$ time. Thus it is easy to see that time complexity is $O(1)$ in both cases.

(b) $N_{>i}\left(v_{i}\right) \subset C\left(v_{j}\right)$ and there are no vertex sets in $N_{>i}\left(v_{i}\right)$ : We remove $N_{>i}\left(v_{i}\right)$ from $C\left(v_{j}\right)$ and make a new vertex set $N_{>i}\left(v_{i}\right)$ shared by $C\left(v_{j}\right)$ and $C_{M}\left(v_{i}\right)=\left\{v_{i}\right\} \cup N_{>i}\left(v_{j}\right)$. We can observe that $N_{>i}\left(v_{i}\right) \subset C\left(v_{j}\right)$ and there are no vertex sets in $N_{>i}\left(v_{i}\right)$ if and only if $\left|N_{>i}\left(v_{i}\right)\right|<\left|C\left(v_{j}\right)\right|$ and $\left|\ell\left(C\left(v_{j}\right)\right)\right|=\left|C\left(v_{j}\right)\right|$. Thus, CliqueLaminarTree recognizes this case in $O(1)$ time, and handles it in case (3). It is easy to see that case (3) can be done in $O\left(\left|N_{>i}\left(v_{i}\right)\right|\right)=O\left(\operatorname{deg}\left(v_{i}\right)\right)$ time. We note that, in the case, we do not mind if $C\left(v_{j}\right)$ is maximal or not. In any case, the property does not change for $C\left(v_{j}\right)$.

(c) $N_{>i}\left(v_{i}\right) \subset C\left(v_{j}\right)$ and there are vertex sets $L_{1} \subset L_{2} \subset \cdots \subset L_{h} \subset N_{>i}\left(v_{i}\right)$ : We first observe that the nodes $L_{1}, L_{2}, \ldots, L_{h}$, and $C\left(v_{j}\right)$ form a directed path in $\vec{T}$ in the case. (Hence we can recognize this case in $O\left(\left|N_{>i}\left(v_{i}\right)\right|\right)=O\left(\operatorname{deg}\left(v_{i}\right)\right)$ time, which will be used in Theorem 12.) Thus we make a new vertex set $L:=N_{>i}\left(v_{i}\right)$ with $\ell(L)=N_{>i}\left(v_{i}\right) \backslash L_{h}$. The set $N_{>i}\left(v_{i}\right) \backslash L_{h}$ is given by $N_{>i}\left(v_{i}\right) \cap \ell\left(C\left(v_{j}\right)\right)$. Then we update $\ell\left(C\left(v_{j}\right)\right)$ by $\ell\left(C\left(v_{j}\right)\right) \backslash N_{>i}\left(v_{i}\right)$. It is easy to add a maximal clique $C_{M}\left(v_{i}\right)=\left\{v_{i}\right\} \cup N_{>i}\left(v_{i}\right)$. Next, we have to update arcs around $C\left(v_{j}\right)$. By Lemma 11, this process is simple; we can find $L_{h}$ in $O\left(\operatorname{deg}\left(v_{i}\right)\right)$ 
time, and there are no other vertex set $L^{\prime}$ that has an $\operatorname{arc}\left(L^{\prime}, C\left(v_{j}\right)\right)$ which has to be updated. We note that there can be some vertex set $L^{\prime}$ with an $\operatorname{arc}\left(L^{\prime}, C\left(v_{j}\right)\right)$. But $L^{\prime}$ is independent from $L$ in this case, and hence we do not have to mind it. Finally, we change the $\operatorname{arc}\left(L_{h}, C\left(v_{j}\right)\right)$ to $\left(L_{h}, L\right)$, and add the $\operatorname{arcs}\left(L, C\left(v_{j}\right)\right)$ and $\left(L, C_{M}\left(v_{i}\right)\right)$. Therefore the time complexity in the last case is $O\left(\operatorname{deg}\left(v_{i}\right)\right)$ time.

By the above case analyses, Theorem 9 is settled.

\section{Applications of Clique Laminar Trees}

\subsection{The Recognition Problem}

Theorem 12 The recognition problem for ptolemaic graphs can be solved in linear time.

Proof.(Sketch.) Using the LBFS, we can obtain a perfect elimination ordering of $G$ in linear time if $G$ is chordal (and reject it if $G$ is not chordal). For a chordal graph, we run modified CliQUELAMINARTREE. It is not difficult to modify CliqueLAminarTReE to reject it if $G$ is not distance hereditary. The key fact is that, if $G$ is ptolemaic, $N_{>i}\left(v_{i}\right)$ corresponds to a maximal directed path in $\vec{T}(\mathcal{C}(G))$ as follows; suppose that we have vertex sets $L_{1} \subset L_{2} \subset \cdots \subset L_{h} \subset N_{>i}\left(v_{i}\right) \subset C\left(v_{j}\right)$ in case (c) in the proof of Theorem 9. In this case, (1) the nodes $L_{1}, L_{2}, \ldots, L_{h}, C\left(v_{j}\right)$ form a (connected) directed path in $T(\mathcal{C}(G)),(2)$ there are no other set $L$ with $L \subset L_{1},(3)$ all vertices in $L_{h}$ (and hence $L_{1} \cup L_{2} \cup \cdots \cup L_{h}$ ) belong to $N_{>i}\left(v_{i}\right)$, and (4) some vertices in $C\left(v_{j}\right)$ may not be in $N_{>i}\left(v_{i}\right)$. Checking them can be done in $O\left(\left|N_{>i}\left(v_{i}\right)\right|\right)=O\left(\operatorname{deg}\left(v_{i}\right)\right)$ time for each $i$, and otherwise, the vertex sets in the tree are not laminar, and hence it would be rejected. Cases (a) and (b) can be seen as special cases of case (c). Therefore, the total running time of the modified CliqueLAMINARTREE is still $O(n+m)$.

We note that Theorem 12 is not new. Since a graph is ptolemaic if and only if it is chordal and distance-hereditary [16], distance hereditary graphs are recognized in linear time [14, 9, 4], and chordal graphs are also recognized in linear time $[22,24]$, we have the result by combining them. We dare to state Theorem 12 to show that we can recognize if a graph is ptolemaic and then construct its clique laminar tree at the same time in linear time, and the algorithm is much simpler and more straightforward than the combination of known algorithms. (As noted in Introduction, the linear time algorithm for recognition of distance hereditary graphs is not so simple.)

\subsection{The Graph Isomorphism Problem}

Theorem 13 The graph isomorphism problem for ptolemaic graphs can be solved in linear time.

Proof. Given a ptolemaic graph $G=(V, E)$, the labeled clique laminar tree $\vec{T}(\mathcal{C}(G))$ is uniquely determined up to isomorphism by maximal cliques. Each vertex in $V$ appears once in $\vec{T}(\mathcal{C}(G))$, and the number of nodes in $\vec{T}(\mathcal{C}(G))$ is at most $2|V|-1$ by Lemma 3 . Thus the representation of $\vec{T}(\mathcal{C}(G))$ requires $O(|V|)$ space. The graph isomorphism problem for labeled trees can be done in linear time (see, e.g., [19]), which completes the proof.

\subsection{The Hamiltonian Cycle Problem}

We assume that a ptolemaic graph $G=(V, E)$ is given by a directed clique laminar tree $\vec{T}(\mathcal{C}(G))=$ $(\mathcal{C}(G), A(G))$. Then the main theorem in this section is the following:

Theorem 14 The Hamiltonian cycle problem for ptolemaic graphs can be solved in $O(n)$ time.

We remind that $\vec{T}(G)$ takes $O(n)$ space. We first observe that if $\vec{T}(G)$ contains a vertex set $C$ with $|C|=1$, the vertex in $C$ is a cutpoint of $G$, and hence $G$ does not have a Hamiltonian cycle. This condition can be checked in $O(n)$ time over $\vec{T}(G)$. Hence, hereafter, we assume that $G$ has no cutpoint, or equivalently, any vertex set $C$ in $\mathcal{C}$ satisfies $|C|>1$.

Let $L$ be a vertex set in $\mathcal{C}(G)$. Each vertex set $L^{\prime}$ with $\left(L, L^{\prime}\right) \in A(G)$ is said to be a child of $L$, and each vertex set $L^{\prime \prime}$ with $\left(L^{\prime \prime}, L\right) \in A(G)$ is said to be a parent of $L$. That is, a child $L^{\prime}$ and a parent $L^{\prime \prime}$ of $L$ satisfy $L^{\prime \prime} \subset L \subset L^{\prime}$. We define ancestors and descendants for $L$ as in ordinary trees. Note here that any node $L$ in $\vec{T}(G)$ is an ancestor and descendant of itself. We denote by $c(L)$ and $p(L)$ the number of 


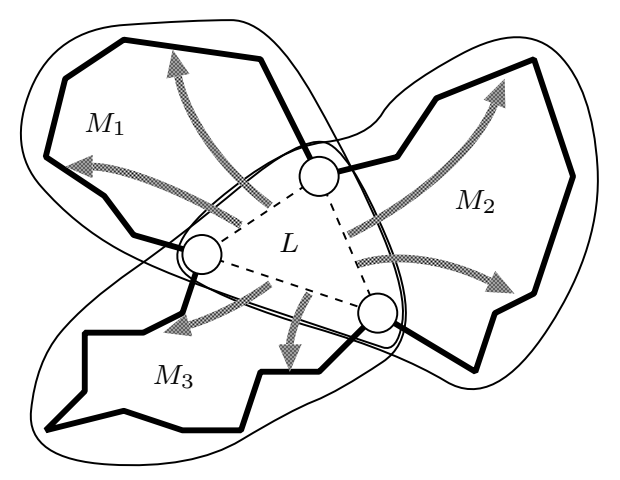

Figure 3: Assignment of an edge to a path.

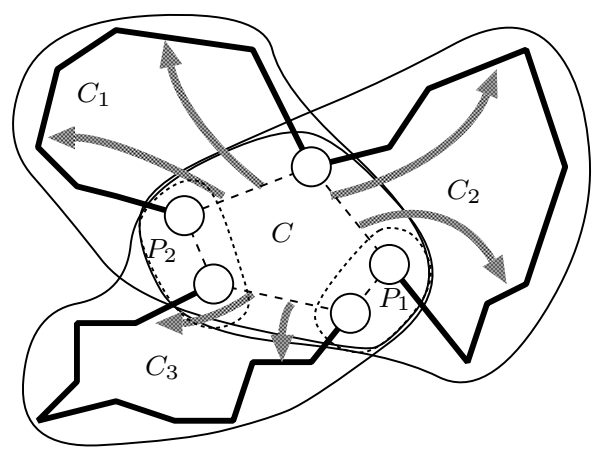

Figure 4: Connection of children and parents.

children of $L$ and the number of parents of $L$ in $\vec{T}(G)$, respectively. Hence $c(M)=0$ for each maximal clique $M$, and $p(L)=0$ for each minimal vertex set $L$.

We first consider a minimal vertex set $L$ with $p(L)=0$. By Lemma 5 , each $L$ in $\mathcal{L}(G)$ is a separator of $G$. It is not difficult to see that if we remove $L$ from $G$, we have $c(L)$ connected components. Hence, if $|L|<c(L), G$ cannot have a Hamiltonian cycle. On the other hand, when $|L|=c(L)$, any Hamiltonian cycle uses all vertices in $L$ to connect each connected components. This fact can be seen as follows (Figure 3); we first make a cycle of length $|L|$ in $L$, and next replace each edge by a path through the vertices in one vertex set corresponding to a child of the node $L$. We say that we assign each edge to distinct child of $L$. (When $|L|=2$, we temporarily assign two (multi)edges.) If $|L|>c(L)$, we can construct a Hamiltonian cycle that uses $|L|-c(L)$ edges in $G[L]$. In this case, we need to assign $c(L)$ edges in $L$ to construct a cycle, and we also have $|L|-c(L)$ edges which can be assigned in some other descendants. We then define the margin $m(L)$ by $|L|-c(L)=|\ell(L)|-c(L)$. That is, if $m(L)<0, G$ has no Hamiltonian cycle, and if $m(L)>0$, we have $m(L)$ edges in $L$ which can be assigned in some descendants. We note that a margin can be inherited only from an ancestor to an descendant.

We here define a distribution $\delta\left(\left(C_{i}, C_{j}\right)\right)$ of the margin, which is a function assigned to each arc $\left(C_{i}, C_{j}\right) \in \vec{T}(\mathcal{C} G)$. Let $C_{1}, \ldots, C_{c(L)}$ be the children of $L$. Then for $i=1,2, \ldots, c(L)$ each $\operatorname{arc}\left(L, C_{i}\right)$ has a distribution $\delta\left(\left(L, C_{i}\right)\right)$ with $\sum_{i=1}^{c(L)} \delta\left(\left(L, C_{i}\right)\right)=m(L)$. That is, each child $C_{i}$ inherits $\delta\left(\left(L, C_{i}\right)\right)$ margins from $L$, and some descendants of $C_{i}$ will consume $\delta\left(\left(L, C_{i}\right)\right)$ margins from $L$. The way to compute the distribution will be discussed later.

We next consider a vertex set $C$ with $p(C)>0$ and $c(C) \geq 0$, that is, $C$ is a vertex set which is not minimal. Let $P_{1}, P_{2}, \ldots, P_{h}$ be parents of $C$ and $C_{1}, C_{2}, \ldots, C_{k}$ children of $C$ in $\vec{T}(G)$. That is, we have $P_{i} \subset C \subset C_{j}$ for each $i$ and $j$ with $1 \leq i \leq h=p(C)$ and $1 \leq j \leq k=c(C)(k=c(C)=0$ when $C$ is maximal clique). We assume that $m\left(P_{i}\right)$ and $\delta\left(\left(P_{i}, C\right)\right)$ are already defined for each $P_{i}$, and $m\left(P_{i}\right) \geq 0$ (otherwise $G$ does not have any Hamiltonian cycle). As in case (1), we have to assign $c(L)$ edges in $C$. In the case, each parent $P_{i}$ can be used as a single vertex if $\delta\left(\left(P_{i}, C\right)\right)=0$ (Figure 4); we first cut (remove) the assigned edge in $P_{i}$ for $C$, and replace it by the path through all vertices in $C$ and its children. If $\delta\left(\left(P_{i}, C\right)\right)>0$ for some $P_{i}$, we can use the additional vertices to connect children $C_{j}$. Hence the margin $m(C)$ is defined by $|\ell(C)|+h+\sum_{i=1}^{h} \delta\left(\left(P_{i}, C\right)\right)-k=|\ell(C)|+\sum_{i=1}^{h}\left(\delta\left(\left(P_{i}, C\right)\right)+1\right)-k$. The distribution of the margin is defined as the same as in $(1) ; \delta\left(\left(C, C_{i}\right)\right)$ is a function such that $\sum_{i=1}^{k} \delta\left(\left(C, C_{i}\right)\right)=m(C)$.

Above discussion leads us to the following theorem:

Theorem 15 Let $G=(V, E)$ be a ptolemaic graph. Then $G$ has a Hamiltonian cycle if and only if there exist feasible distributions of margins such that each vertex set $C$ in $\mathcal{C}$ satisfies $m(C) \geq 0$.

It is easy to see that the margin $m(M)$ for any maximal clique $M$ is positive in case (2) since $k=0$. In other words, each maximal clique $M$ does not require any distribution of margins from its parents.

Our linear time algorithm, say $\mathcal{A}$, runs on $T(G) ; \mathcal{A}$ collects the leaves in $T(G)$, computes the margins, and repeats this process by computing the margin of $C$ such that all neighbors of $C$ have been processed except exactly one neighbor. The precise procedure for each vertex set $C$ is described as follows:

(1) When the vertex set $C$ is a leaf of $T(G), C$ is a maximal clique in $G$, and hence $\delta((P, C))$ is set to 0 , where $P$ is the unique parent of $C$. 

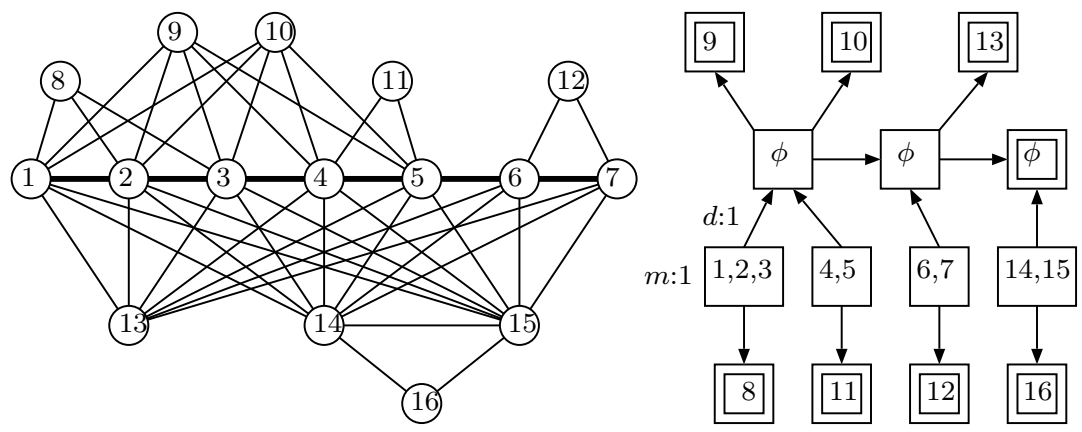

Figure 5: Definition of margins.

(2) When $C$ is not a leaf of $T(G)$, let $P_{1}, P_{2}, \ldots, P_{h}$ be parents of $C$ in $\vec{T}(G), C_{1}, C_{2}, \ldots, C_{k}$ children of $C$ in $\vec{T}(G)$, and $X$ be the only neighbor which is not processed. Without loss of generality, we assume that either $X=P_{h}$ or $X=C_{k}$. To simplify the notation, we define $h^{\prime}=h-1$ and $k^{\prime}=k$ if $X=P_{h}$, and $h^{\prime}=h$ and $k^{\prime}=k-1$ if $X=C_{k}$. We have three subcases.

(a) If $C$ is a maximal clique in $G$, or $k=0, C$ requires no distribution of margins. Hence, $\mathcal{A}$ assigns $\delta((X, C))=0($ since $X \subset C)$.

(b) If $C$ is a minimal vertex set with $k>0, h=0$, we have $X=C_{k}$. Then $\mathcal{A}$ first computes $m(C)=$ $|\ell(C)|-k^{\prime}$. Then, for each $i$ with $i=1,2, \ldots, k^{\prime}$, each child $C_{i}$ has been processed, and it requires distribution $\delta\left(\left(C, C_{i}\right)\right)$ to $C$. Hence $\mathcal{A}$ computes $\delta^{\prime}((C, X))=m(C)-\sum_{i=1}^{k^{\prime}} \delta\left(\left(C, C_{i}\right)\right)=|\ell(C)|-$ $\sum_{i=1}^{k^{\prime}}\left(\delta\left(\left(C, C_{i}\right)\right)+1\right)$. If $\delta^{\prime}((C, X))<0, G$ has no Hamiltonian cycles. Otherwise, $\mathcal{A}$ sets $\delta((C, X)):=$ $\delta^{\prime}((C, X))$.

(c) When $C$ is not a maximal clique with $k>0$ and $h>0, \mathcal{A}$ first computes the margin $m(C)=$ $|\ell(C)|+\sum_{i=1}^{h^{\prime}}\left(\delta\left(\left(P_{i}, C\right)\right)+1\right)-k^{\prime}$. Next, $\mathcal{A}$ distributes the margin $m(C)$ to the children $C_{1}, \ldots, C_{k^{\prime}}$ by computing $\delta^{\prime}:=m(C)-\sum_{i=1}^{k^{\prime}} \delta\left(\left(C, C_{i}\right)\right)=|\ell(C)|+\sum_{i=1}^{h^{\prime}}\left(\delta\left(\left(P_{i}, C\right)\right)+1\right)-\sum_{i=1}^{k^{\prime}}\left(\delta\left(\left(C, C_{i}\right)\right)+1\right)$. The value $\delta^{\prime}$ indicates the margin that will be exchanged between $C$ and $X$.

If $X=C_{k}$, that is, $(C, X)$ is the $\operatorname{arc}$ in $\vec{T}(G), \mathcal{A}$ distributes all margins $\delta^{\prime}$ to $X$, or sets $\delta((C, X))=\delta^{\prime}$. The margin can be inherited from a parent to a child. Thus, in this case, if $\delta^{\prime}<0, G$ has no Hamiltonian cycles. When $\delta^{\prime} \geq 0, \mathcal{A}$ will use the margin $\delta^{\prime}$ when it processes the vertex set $X$.

On the other hand, if $X=P_{h}$, that is, $(X, C)$ is the arc in $\vec{T}(G)$, the margin will be distributed from $X$ to $C$. Hence, if $\delta^{\prime}<0$, the vertex $C$ borrows margin $\delta^{\prime}$ from $X$ which will be adjusted when the vertex $X$ is chosen by $\mathcal{A}$. Thus $\mathcal{A}$ sets $\delta((X, C))=-\delta^{\prime}$ in this case. If $\delta^{\prime} \geq 0$, the margin is useless since the parent $X$ only counts the number of its children $C$, and does not use their margins. Therefore, $\delta((X, C))$ will never referred, and hence $\mathcal{A}$ does nothing.

(3) When $C$ is the last node of the process; that is, every value of $\delta\left(\left(C, C^{\prime}\right)\right)$ or $\delta\left(\left(C^{\prime}, C\right)\right)$ for each neighbor $C^{\prime}$ of $C$ has been computed. Let $P_{1}, P_{2}, \ldots, P_{h}$ be parents of $C$ in $\vec{T}(G), C_{1}, C_{2}, \ldots, C_{k}$ children of $C$ in $\vec{T}(G)$. In the case, $\mathcal{A}$ computes $m(C)=|\ell(C)|+\sum_{i=1}^{h}\left(\delta\left(\left(P_{i}, C\right)\right)+1\right)-\sum_{i=1}^{k}\left(\delta\left(\left(C, C_{i}\right)\right)+1\right)$. If $m(C)<0, C$ does not have enough margin. Hence $G$ has no Hamiltonian cycle. Otherwise, every node has enough margin, and hence $G$ has a Hamiltonian cycle.

A simple example is depicted in Figure 5, where $\{1,2, \ldots, 7\}$ induces a clique; the node $C$ with $\ell(C)=\{1,2,3\}$ has margin 1 , and the arc from $C$ to $C^{\prime}$ with $C^{\prime}=\{1,2,3,4,5\}$ has distribution 1 . The other nodes have margin 0 , and the other arcs have distribution 0 . Hence the graph in Figure 5 has a Hamiltonian cycle, e.g., (1, 8, 2, 9, 3, 10, 4, 11, 5, 14, 16, 15, 7, 12, 6, 13, 1).

The correctness of $\mathcal{A}$ can be proved by a simple induction for the number of nodes in $\vec{T}(G)$ with Theorem 15. On the other hand, since $T(G)$ contains $O(n)$ nodes, the algorithm runs in $O(n)$ time and space, which completes the proof of Theorem 14. We note that the construction of a Hamiltonian cycle can be done simultaneously in $O(n)$ time and space. 


\section{Concluding Remarks}

In this paper, we present new tree representations (data structures) for ptolemaic graphs. The result enables us to use the dynamic programming technique to solve some basic problems on this graph class. We presented a linear time algorithm for the Hamiltonian cycle problem, as one of such typical examples. To develop such efficient algorithms based on the dynamic programming for other problems are future works.

\section{Acknowledgment}

The authors thank to Professor Hiro Ito, who pointed out a flaw in our polynomial time algorithm for finding a longest path in a ptolemaic graph stated in [25]. That motivated us to investigate the problems in this paper.

\section{References}

[1] H.-J. Bandelt and H.M. Mulder. Distance-Hereditary Graphs. Journal of Combinatorial Theory, Series B, 41:182-208, 1986.

[2] A. Brandstädt and F.F. Dragan. A Linear-Time Algorithm for Connected $r$-Domination and Steiner Tree on Distance-Hereditary Graphs. Networks, 31:177-182, 1998.

[3] A. Brandstädt, V.B. Le, and J.P. Spinrad. Graph Classes: A Survey. SIAM, 1999.

[4] A. Bretscher, D. Corneil, M. Habib, and C. Paul. A Simple Linear Time LexBFS Cograph Recognition Algorithm. In Graph-Theoretic Concepts in Computer Science (WG 2003), pages 119-130. Lecture Notes in Computer Science Vol. 2880, Springer-Verlag, 2003.

[5] H.J. Broersma, E. Dahlhaus, and T. Kloks. A linear time algorithm for minimum fill-in and treewidth for distance hereditary graphs. Discrete Applied Mathematics, 99:367-400, 2000.

[6] M.-S. Chang, S.-Y. Hsieh, and G.-H. Chen. Dynamic Programming on Distance-Hereditary Graphs. In Proceedings of 8th International Symposium on Algorithms and Computation (ISAAC '97), pages 344-353. Lecture Notes in Computer Science Vol. 1350, Springer-Verlag, 1997.

[7] M.-S. Chang, S.-C. Wu, G.J. Chang, and H.-G. Yeh. Domination in distance-hereditary graphs. Discrete Applied Mathematics, 116:103-113, 2002.

[8] D.G. Corneil. Lexicographic Breadth First Search — A Survey. In Graph-Theoretic Concepts in Computer Science (WG 2004), pages 1-19. Lecture Notes in Computer Science Vol. 3353, SpringerVerlag, 2004.

[9] G. Damiand, M. Habib, and C. Paul. A Simple Paradigm for Graph Recognition: Application to Cographs and Distance Hereditary Graphs. Theoretical Computer Science, 263:99-111, 2001.

[10] A. D'Atri and M. Moscarini. Distance-Hereditary Graphs, Steiner Trees, and Connected Domination. SIAM Journal on Computing, 17(3):521-538, 1988.

[11] M. Farber. Independent Domination in Chordal Graphs. Operations Research Letters, 1(4):134-138, 1982.

[12] F. Gavril. Algorithms for Minimum Coloring, Maximum Clique, Minimum Covering by Cliques, and Maximum Independent Set of a Chordal Graph. SIAM Journal on Computing, 1(2):180-187, 1972.

[13] M.C. Golumbic. Algorithmic Graph Theory and Perfect Graphs. Annals of Discrete Mathematics 57. Elsevier, 2nd edition, 2004.

[14] P.L. Hammer and F. Maffray. Completely Separable Graphs. Discrete Applied Mathematics, 27:8599, 1990. 
[15] E. Howorka. A Characterization of Distance-Hereditary Graphs. Quart. J. Math. Oxford (2), 28:417-420, 1977.

[16] E. Howorka. A Characterization of Ptolemaic Graphs. Journal of Graph Theory, 5:323-331, 1981.

[17] R.-W. Hung and M.-S. Chang. Linear-time algorithms for the Hamiltonian problems on distancehereditary graphs. Theoretical Computer Science, 341:411-440, 2005.

[18] P.N. Klein. Efficient Parallel Algorithms for Chordal Graphs. SIAM Journal on Computing, 25(4):797-827, 1996.

[19] J. Köbler, U. Schöning, and J. Torán. The Graph Isomorphism Problem: Its Structural Complexity. Birkhäuser, 1993.

[20] B. Korte and J. Vygen. Combinatorial Optimization, volume 21 of Algorithms and Combinatorics. Springer, 2000.

[21] F. Nicolai and T. Szymczak. Homogeneous Sets and Domination: A Linear Time Algorithm for Distance-Hereditary Graphs. Networks, 37(3):117-128, 2001.

[22] D.J. Rose, R.E. Tarjan, and G.S. Lueker. Algorithmic Aspects of Vertex Elimination on Graphs. SIAM Journal on Computing, 5(2):266-283, 1976.

[23] J.P. Spinrad. Efficient Graph Representations. American Mathematical Society, 2003.

[24] R.E. Tarjan and M. Yannakakis. Simple Linear-Time Algorithms to Test Chordality of Graphs, Test Acyclicity of Hypergraphs, and Selectively Reduce Acyclic Hypergraphs. SIAM Journal on Computing, 13(3):566-579, 1984.

[25] R. Uehara and Y. Uno. Efficient Algorithms for the Longest Path Problem. In 15th Annual International Symposium on Algorithms and Computation (ISAAC 2004), pages 871-883. Lecture Notes in Computer Science Vol.3341, Springer-Verlag, 2004.

[26] H.-G. Yeh and G.J. Chang. Centers and medians of distance-hereditary graphs. Discrete Mathematics, 265:297-310, 2003. 